\title{
PERCEPTUAL-POSTURAL CORRELATION OF VISUAL DEPENDENCE IN HEALTHY ADULTS ACROSS AGE GROUPS
}

\section{Shu-Chun Lee}

Department of Physical Therapy, Shu-Zen College of Medicine and Management, Kaohsiung, Taiwan.

\section{ABSTRACT}

Background: Visual dependence is a term to describe over reliance on vision than other sensory systems on orientation and balance. Perceptual and postural visual dependence have been reported in many articles but usually discussed in separate studies and focused on certain age groups.

Objective: The aims of this study are twofold. The first investigates the correlation of perceptual and postural visual dependence in healthy adults across age groups. The second examines the correlation between age and visual dependence at perceptual and postural levels respectively.

Methods: Forty healthy adults were recruited and assessed for levels of perceptual and postural visual dependence. The former was distinguished through subjective visual vertical (SVV) tilt by Rod and Disc test; the latter was measured by sway acceleration in six sensory conditions with various visual and proprioceptive inputs.

Results: The results showed moderate and positive correlation between the values of SVV tilt and sway acceleration with the subject standing on foam with a visual disturbance applied. A low and positive correlation was also observed between age and the value of SVV tilt and with sway acceleration, where both visual and proprioceptive inputs were altered.

Conclusion: These findings suggest perceptual visual dependence was significantly increased with postural visual dependence in healthy adults across age groups, particularly when performing a challenging balance task. Greater visual dependence at both perceptual and postural levels with increasing age was also found.

KEY WORDS: Correlation, Perception, Balance, Rod And Disc Test, Visual Dependence.

Address for correspondence: Dr. Shu-Chun Lee, PhD, Department of Physical Therapy, Shu-Zen College of Medicine and Management, Kaohsiung, Taiwan. Tel: 886-7-6979-315

Fax: 886-7-6979-398 E-Mail: shu-chun.lee@outlook.com

\begin{tabular}{|l|l|}
\hline \multicolumn{3}{|c|}{ Access this Article online } \\
\hline \multirow{2}{*}{ Quick Response code } & International Journal of Physiotherapy and Research \\
& \multicolumn{1}{|c|}{ ISSN 2321- 1822 } \\
www.ijmhr.org/ijpr.html
\end{tabular}

\section{INTRODUCTION}

Effective orientation and balance control requires the integration of proprioceptive, vestibular and visual sensory systems [1].

However, some people over rely on visual input, i.e. persons who are visually dependent, for spatial orientation and postural responses. Several studies have reported that the level of visual dependence at the perceptual level increased with age, using the method of measuring errors of subjective visual vertical (SVV) induced by visual stimulation [2,3]. Increased visual dependence with age was also observed at the postural level, that older adults had significantly more postural sway in response to optic flow compared to younger adults and showed continued center of pressure oscillation even following the visual disturbance [4-6].

Investigation of visual dependence in most previous studies used younger adults as the con- 
trol group in comparison with older adults $[4,6]$, but there is lack of understanding regarding the middle-age group. In Kobayashi et al.'s study, linear regression of age and SVV tilt was analyzed from 21 to 63 years [3], it was unclear, however, whether values of SVV tilt in older adults over 65 continue increasing with age. Overall, perceptual and postural visual dependence were commonly discussed in separate articles and only focused on certain age groups. Thus, whether a correlation between the two and whether both simultaneously increase with age requires investigation. The primary aim of this study was to investigate the correlation of perceptual and postural visual dependence in healthy adults across age groups. The second was to examine the correlation between age and visual dependence at the perceptual and postural levels respectively.

\section{MATERIALS AND METHODS}

Subjects: Forty healthy adults ( 9 male, range 20-76 yrs.; mean $55 \pm 15$ SD yrs.) were recruited from neighboring communities. All subjects were screened for exclusion criteria including: (1) inability to stand independently without an assistive device; (2) a history of neurological disease including epilepsy, migraine or fainting; (3) a history of vestibular disorders; (4) clinically detectable proprioception loss; (5) uncorrected visual impairment or binocular visual acuity (with corrective lenses) of worse than 20/40; (6) more than one fall in the previous one year; (7) previous exposure to the Rod and Disc Test (RDT). They gave written informed consent to participate in the study, which was approved by the local Ethics Committee.

Protocol: All subjects were assessed for levels of visual dependence at the perceptual and postural level in a counterbalanced order.

Perceptual visual dependence: Perceptual visual dependence was distinguished through assessment of SVV tilt by the RDT [7]. A computerized RDT was displayed on an LCD TV monitor (Model TL-42S3000T, CHIMEI, Taiwan) but the image was rendered circular $(53 \mathrm{~cm}$ diameter) by blackout material. Subjects sat upright on a padded armless chair with their eyes $50 \mathrm{~cm}$ from the screen while their knees were extended and their feet were dorsiflexed so that only the heels were in contact with the floor in order to diminish possible proprioceptive cues from the plantar surface of the feet. The center of the rod (14 cm length) was aligned to the subjects' eye level via adjustment of the chair height. Subjects closed their eyes whilst the rod was deviated either $20^{\circ}$ clockwise (CW) or counter-clockwise (CCW) in a randomized order. They were then asked to open their eyes and align the rod to their SVV at their own pace with florescent discs stationary around the rod, using the wheel of a wireless computer mouse and confirm verbally when the task was completed. This process was then repeated with discs rotating at $30 \% \mathrm{~s}$ in $\mathrm{CW}$ and $\mathrm{CCW}$ directions. Four trials each were performed for the stationary and then rotating (in both directions) disc tests. The image and subject were surrounded by blackout material ensuring complete darkness to minimize any external vertical and horizontal visual reference cues. The RDT software automatically recorded SVV tilt values taken as angular deviations from true gravitational vertical $\left(0^{\circ}\right)$. The tilt of the top of the rod to the subject's right or left was indicated as a positive or negative angle, respectively. Each subject's average SVV tilt angle with the disc stationary was subtracted from those obtained with the rotating disc. Greater SVV tilt $\left({ }^{\circ}\right.$, absolute value) represents a higher level of perceptual visual dependence.

Postural visual dependence: Postural visual dependence was assessed by postural sway acceleration $(\mathrm{m} / \mathrm{s} 2)$, which was measured using a smartphone device (Apple iPhone 4s, Apple Computer Inc., USA) loaded with the SPARKvue Application software (v2.5.0, PASCO Scientific, USA). The iPhone device was firmly attached to the subject by means of an elastic belt around the waist over the middle part of the lower back over the L3 (3rd lumbar) vertebra process so that it was attached close to the center of gravity (COG) of the human body [8]. Several studies have indicated that accelerometric mobile devices, such as an iPhone or iPod, showed the capacity to quantify balance parameters with accuracy and reliability [9] when compared to laboratory equipment such as strain gauges and accelerometers. Therefore, the SPARKvue Application software paired with 
a smartphone device can be relied on as a tool to measure postural sway through a tri-axial accelerometer [10].

Subjects stood with their hands at their sides, bare feet together, and performed 6 sensory conditions with various visual cues including Eyes open (EO): subjects stood looking at the static RDT image; Eyes closed (EC): subjects stood quietly with both eyes closed; Visual disturbance: subjects stood while looking at the RDT image with discs rotating at $30 \% \mathrm{~s}$ in a CW direction. The subjects were also evaluated on varying supporting conditions including firm and foam (Airex Elite Non-slip Foam Balance Pad, USA) surfaces. Together, these six conditions alter the availability and accuracy of visual and proprioceptive inputs for postural control (C1C6; Table 1). Each condition was repeated once and conducted in a randomized order. A researcher stood closely behind the subject during all trials for safety reasons. Between trials, each subject received a 10 second rest, during which subjects were able to freely move and walk around.

Postural sway was recorded for 20 seconds with a sample frequency of $100 \mathrm{~Hz}$ in $x$ (mediallateral) and y (anterior-posterior) directions under all conditions; the middle 10 second segment of each sample was analyzed in order to avoid postural adjustments and fatigue at the beginning and the end of the task. All the data were exported from the SPARKvue Application software to Microsoft Excel $^{\circledR}$ (Version 2010, Windows 7) for analysis. Sway acceleration was defined as the average change of the COG velocity with respect to time in the horizontal plane and reflects postural sway. Mean sway acceleration in all conditions were normalized to baseline (C1). Greater sway acceleration in C2, C3, C5 and C6 reflect the greater influence of vision on balance, indicating higher level of postural visual dependence.

Statistical analysis: All data are presented as mean \pm standard deviation (SD). SPSS (Version 19.0. Armonk, NY: IBM Corp.) was used for all statistical analysis with significance assumed at $p<0.05$. The Shapiro-Wilk test was used to analyze all data to determine if it was normally distributed. All parameters were not normallydistributed except sway acceleration in $\mathrm{C} 3$ and
C6 conditions. Thus, the Spearman rank correlation test was performed to examine the correlation between value of SVV tilt and sway acceleration in all conditions respectively. The same test was used again for the correlation of age and value of SVV tilt or sway acceleration in all conditions. It was assumed that the Spearman's correlation coefficient $(\mathrm{rs})<0.3$ is very low; $0.3-0.5$ low; $0.5-0.7$ moderate; $0.7-0.9$ high and $>0.9$ very high [11].

\section{RESULTS}

There was an only a moderate positive correlation between the value of SVV tilt and sway acceleration in C6 ( $r=0.595, p<0.001$; Fig. 1$)$. A low positive correlation was observed on age with value of SVV tilt ( $r=0.389, p=0.013$; Fig. $2)$ and with sway acceleration in $\mathrm{C} 6(r=0.372$, $\mathrm{p}=0.018$; Fig. 3).

Table 1: Postural visual dependence was assessed in six sensory conditions with three visual and two supporting conditions.

\begin{tabular}{|c|c|c|c|c|}
\hline & & \multicolumn{3}{|c|}{ Visual conditions } \\
\hline & & EO & EC & $\begin{array}{c}\text { Visual } \\
\text { disturbance }\end{array}$ \\
\hline $\begin{array}{l}\text { Supporting } \\
\text { conditions }\end{array}$ & Firm surface & C1 & C2 & C3 \\
\cline { 2 - 5 } & Foam surface & C4 & C5 & C6 \\
\hline
\end{tabular}

$\mathrm{EC}=$ eyes closed; $\mathrm{EO}=$ eyes open.

Fig. 1: There is a positive moderate correlation $(r=0.595$, $p<0.001$ ) of the values of SVV tilt and sway acceleration in $\mathrm{C6}$.

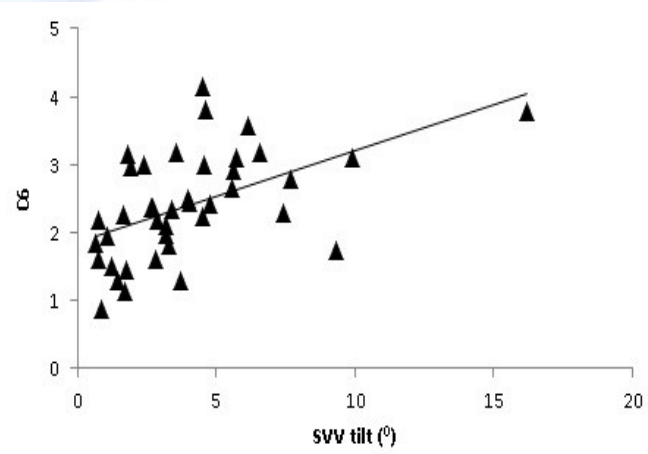

Fig. 2: Scatter graph shows a positive low correlation ( $r$ $=0.389, p=0.013$ ) of age and the values of SVV tilt.

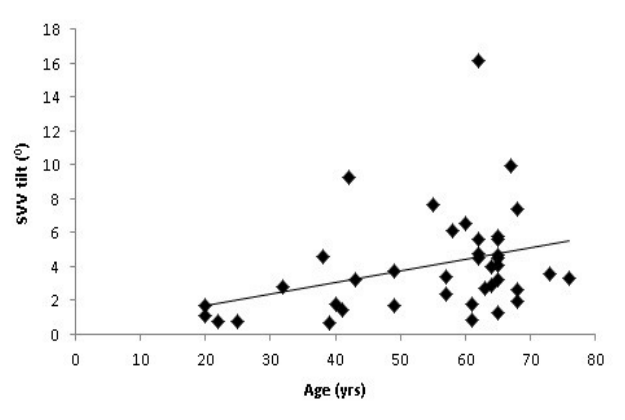


Fig. 3: It represents a positive low correlation $(r=0.372$, $p=0.018$ ) of age and the sway acceleration in C6.

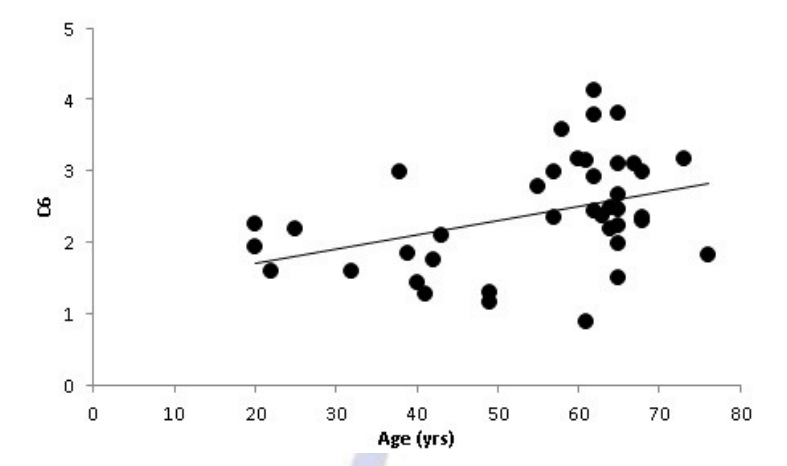

\section{DISCUSSION}

The primary aim of this study was to investigate the correlation of perceptual and postural visual dependence in healthy adults across age groups. The second was to examine the correlation between age and visual dependence at the perceptual and postural levels respectively. The main finding indicates that perceptual visual dependence was significantly increased with postural visual dependence when maintaining a challenging balance task in healthy young, middle-age and older adults. Greater visual dependence at both perceptual and postural levels with increasing age was also found in the current study.

Only significantly positive correlation was found between the value of SVV tilt and sway acceleration in C6, indicating perceptual visual dependence increased with postural visual dependence in healthy adults across age groups. Similar results were found in previous studies [1214].

Pavlou et al. found the positive correlation of the value of SVV tilt measured by the Rod and Frame test (RFT) and the score in a Sensory Organization Test in which both visual and proprioceptive inputs were altered [12]. Isableu et al. reported that visually dependent subjects, distinguished by the RFT, showed instability toward the direction in which the frame was tilted when they were looking at it and standing in the Sharpened Romberg position [12,13]. This result of correlation was not surprising since posture can be influenced by inaccurate orientation [15]. Perceptual orientation is based on the integration of visual, vestibular and proprioceptive sensory systems and postural control requires a complex interaction of musculoskeletal and sensory systems [13]. A tilted or inappropriate internal representation of verticality results in a postural alignment incongruent with respect to gravity, which can lead to imbalance [14]. Taken together, whether static (such as a tilted frame) [12-14] or dynamic (such as rotating discs) visual stimulation, it could affect spatial orientation as well as postural control; although the latter is considered to have a greater influence than the former [15].

Perceptual visual dependence may be a predictor of postural visual dependence because of moderate correlation between the two. Individuals who over-rely on visual input may experience difficulty in resolving situations where visual information is complex or inaccurate, such as crowded or busy environments [16]. This could lead to instability and falling. Through the assessment of perceptual visual dependence, there is a possibility that individuals at high risks of imbalance and even falls can be screened and identified. Evaluation of perceptual visual dependence such as the RFT or RDT is relatively simpler to perform, quicker to measure and easier to interpret compared with the postural visual dependence measured by balance tests. However, the degree of prediction ability still requires further investigation.

The correlation of perceptual and postural visual dependence was only found in $\mathrm{C} 6$ rather than the rest of the conditions. Generally speaking, visually dependent people tended to show imbalance in conditions in which visual cues were removed or made inaccurate [17] Sometimes standing in the presence of visual motion had an even greater destabilizing effect on balance compared to standing with eyes closed [5]. If standing in the absence of reliable proprioceptive cues, inaccurate or unreliable visual cues evoke more exaggerated disorientation and imbalance $[18,19]$. That could explain the only correlation being found in $\mathrm{C} 6$, standing in a foam with visual disturbance, rather than other conditions.

C6 compared to the rest of the conditions is a very challenging balance task. That the correlation was only found in a difficult task could be due to the fact that participants recruited in the study discussed here were healthy adults and 
the majority of them had relatively low levels of perceptual visual dependence when compared to the subjects of previous studies [2,3]. Visually dependent individuals such as patients with vestibular deficits who had greater values of SVV tilt had abnormally large postural responses to a visual stimulation even though they were standing with both legs on a firm surface $[16,20]$. However, in healthy adults, balancing tasks such as standing with both visual and proprioceptive disturbances [12] or in a sharpened Romberg position $[12,13]$ may be difficult enough to challenge visual dependence.

This was the first study to simultaneously investigate perceptual and postural visual dependence in one study and across age groups from 20 to 76 years of age, and the findings indicate greater visual dependence at both perceptual and postural levels with increasing age. However, the correlation was low especially when age increases. A greater inter-individual difference in older adults was observed from scatter graphs. In fact multiple studies report that balance degeneration with age is not necessarily linear [21-23]. Some older subjects had similar or even lower SVV tilt values than younger ones $[5,6,24]$. Although all participants were screened to identify healthy subjects, some may have sub-clinical or undiagnosed sensory impairment $[1,3,25]$ that may affect the levels of visual dependence. All these may be the reasons of low correlation.

\section{CONCLUSION}

Levels of visual dependence were increased simultaneously on both spatial orientation and postural control in healthy adults across age groups, particularly when performing a challenging balance task. Greater visual dependence at either perceptual or postural levels with increasing age was also found in this study.

\section{ABBREVIATIONS}

$$
\begin{aligned}
& \text { CW - Clockwise } \\
& \text { CCW - Counter-clockwise } \\
& \text { COG - Center of gravity } \\
& \text { EC - Eyes closed } \\
& \text { EO - Eyes open } \\
& \text { RDT - Rod and Disc Test } \\
& \text { SWV - Subjective visual vertical } \\
& \text { RFT - Rod and Frame test }
\end{aligned}
$$

\section{Conflicts of interest: None}

\section{REFERENCES}

[1]. Peterka RJ. Sensorimotor integration in human postural control. J Neurophysiol. United States 2002;88(3):1097-118.

[2]. Lord SR, Webster IW. Visual field dependence in elderly fallers and non-fallers. Int J Aging Hum Dev 1990;31(4):267-77.

[3]. Kobayashi H, Hayashi $Y$, Higashino K, Saito A, Kunihiro T, Kanzaki J, et al. Dynamic and static subjective visual vertical with aging. Auris Nasus Larynx 2002;29(4):325-8.

[4]. Wade MG, Lindquist R, Taylor JR, Treat-JacobsonD. Optical flow, spatial orientation, and the control of posture in the elderly. J Gerontol B Psychol Sci Soc Sci 1995;50(1):P51-8.

[5]. Sundermier L, Woollacott MH, Jensen JL, Moore S. Postural sensitivity to visual flow in aging adults with and without balance problems. J Gerontol A Biol Sci Med Sci 1996;51(2):M45-52.

[6]. Borger LL, Whitney SL, Redfern MS, Furman JM. The influence of dynamic visual environments on postural sway in the elderly. J Vestib Res 1999;9(3):197205.

[7]. Held R, Dichigans J, Bauer J. Characteristics of moving visual scenes influencing spatial orientation. Vision Res 1975;15(3):357-65.

[8]. Staab W, Hottowitz R, Sohns C, Sohns JM, Gilbert F, Menke J, et al. Accelerometer and gyroscope based gait analysis using spectral analysis of patients with osteoarthritis of the knee. J Phys Ther Sci 2014;26(7):997-1002.

[9]. Patterson JA, Amick RZ, Thummar T, Rogers ME. Validation of measures from the smartphone sway balance application: a pilot study. Int J Sports Phys Ther 2014;9(2):135-9.

[10]. Briggle J. Analysis of pendulum period with an iPod touch/iPhone. Phys Educ 2013;48(3):285.

[11]. Mukaka MM. Statistics corner: A guide to appropriate use of correlation coefficient in medical research. Malawi Med J 2012;24(3):69-71.

[12]. Pavlou M, Davies RA, Bronstein AM. The assessment of increased sensitivity to visual stimuli in patients with chronic dizziness. J Vestib Res 2006;16(4-5):223-31.

[13]. Shumway-Cook A, Horak FB. Assessing the influence of sensory interaction of balance. Suggestion from the field. Phys Ther 1986;66(10):1548-50.

[14]. Horak FB. Postural orientation and equilibrium: what do we need to know about neural control of balance to prevent falls? Age Ageing 2006;35(Suppl 2):7-11.

[15]. Amblard B, Cremieux J, Marchand AR, Carblanc A. Lateral orientation and stabilization of human stance: static versus dynamic visual cues. Exp brain Res 1985;61(1):21-37.

[16]. Guerraz M, Yardley L, Bertholon P, Pollak L, Rudge P, Gresty $M A$, et al. Visual vertigo: symptom assess- 
ment, spatial orientation and postural control.Brain 2001;124(Pt 8):1646-56.

[17]. Shumway-Cook A, Horak FB. Rehabilitation strategies for patients with vestibular deficits. Neurol Clin 1990;8(2):441-57.

[18]. Golomer E, Cremieux J, Dupui P, Isableu B, Ohlmann T. Visual contribution to self-induced body sway frequencies and visual perception of male professional dancers. Neurosci Lett 1999;267(3):189-92.

[19]. Davlin-Pater C. The effects of visual information and perceptual style on static and dynamic balance. Motor Control 2010;14(3):362-70.

[20]. Bronstein AM. Visual vertigo syndrome: clinical and posturography findings. J Neurol Neurosurg Psychiatry 1995;59(5):472-6.

[21]. Jeka J, Allison L, Saffer M, Zhang Y, Carver S, Kiemel T. Sensory reweighting with translational visual stimuli in young and elderly adults: the role of statedependent noise. Exp brain Res 2006;174(3):517-
[22]. Lazarus NR, Harridge SDR. Exercise, physiological function, and the selection of participants for aging research. J Gerontol A Biol Sci Med Sci 2010;65(8):854-7.

[23]. Kinsella-Shaw JM, Harrison SJ, Colon-Semenza C, Turvey MT. Effects of visual environment on quiet standing by young and old adults. J Mot Behav 2006;38(4):251-64.

[24]. Teasdale N, Stelmach GE, Breunig A. Postural sway characteristics of the elderly under normal and altered visual and support surface conditions. J Gerontol 1991;46(6):B238-44.

[25]. Bronstein AM, Yardley L, Moore AP, Cleeves L. Visually and posturally mediated tilt illusion in Parkinson's disease and in labyrinthine defective subjects. Neurology 1996;47(3):651-6.

How to cite this article:

Shu-Chun Lee. PERCEPTUAL-POSTURAL CORRELATION OF VISUAL DEPENDENCE IN HEALTHY ADULTS ACROSS AGE GROUPS. Int J Physiother Res 2017;5(3):2045-2050. DOI: 10.16965/ijpr.2017.147 